\title{
Perfil motor de crianças quilombolas da comunidade de Tiningú em Santarém-PA
}

\author{
Motor profile of quilombola children in the community of Tiningú in Santarém-PA \\ Perfil motor de niños quilombola en la comunidad de Tiningú en Santarém-PA
}

Recebido: 11/10/2021 | Revisado: 17/10/2021 | Aceito: 18/10/2021 | Publicado: 20/10/2021

\author{
Alexandria da Silva Torres \\ ORCID: https://orcid.org/0000-0001-6556-4374 \\ Centro Universitário da Amazônia, Brasil \\ E-mail: xandriatorres0304vtx@gmail.com \\ Izabela Sandy Sanches Pina \\ ORCID: https://orcid.org/0000-0001-9591-5075 \\ Centro Universitário da Amazônia, Brasil \\ E-mail: izasandy.1998@gmail.com \\ Joice Bastos Lima \\ ORCID: https://orcid.org/0000-0003-1671-5740 \\ Centro Universitário da Amazônia, Brasil \\ E-mail: joycebastos.72@gmail.com \\ Assis Júnior Cardoso Pantoja \\ ORCID: https://orcid.org/0000-0002-8301-3135 \\ Universidade do Estado do Pará, Brasil \\ E-mail: assisfisio_jr@hotmail.com \\ Rafaela dos Santos Reis \\ ORCID: https://orcid.org/0000-0003-0127-0072 \\ Universidade do Estado do Pará, Brasil \\ E-mail: rafaelaibef@gmail.com
}

\begin{abstract}
Resumo
Analisar o desenvolvimento motor é fundamental para estabelecer mudanças no comportamento referente à idade, crescimento, postura, movimento da criança, e à maturação do mecanismo e sistemas do organismo. Em vista disso, é necessária a avaliação do desenvolvimento motor em crianças em comunidade quilombola, pois permite que profissionais da área possam investigar aspectos e características relativos ao desenvolvimento desse público. O objetivo deste estudo foi analisar o perfil motor de crianças quilombolas de Tiningú, em Santarém /PA. Á metodologia, trata-se de uma pesquisa descritiva e exploratória com caráter quantitativo e qualitativo. A amostra foi composta por 18 crianças de ambos os sexos. A ferramenta de coleta dos dados foi a bateria de testes de EDM infantil proposta por Rosa Neto (2002), foi necessário dividir em 2 dias de coleta de dados. Os dados passaram por análises estatísticas descritivas simples no Microsoft Office Excel 2010 e BioEstat versão 5.0. Os resultados, têm-se as classificações da QMG das 18 crianças, aonde 11 crianças obtiveram $61.11 \%$, estando na classificação normal médio, 2 crianças alcançaram $11.11 \%$, apresentando-se compatível com a IC, e 5 crianças atingiram 27,78\%, estando normal baixo. Nenhuma apresentou idade motora superior, inferior e muito inferior. Concluiu-se, a partir dos resultados obtidos, que o desenvolvimento motor das crianças avaliadas, encontra-se dentro dos parâmetros da normalidade, ou seja, compatíveis com a idade cronológica em que elas se encontravam. Diante disso houve 2 grupos de resposta significativa, crianças que se mostraram acima e abaixo da normalidade.
\end{abstract}

Palavras-chave: Desenvolvimento motor; Brincar; Terceira infância.

\begin{abstract}
Analyzing motor development is essential to establish changes in behavior related to age, growth, posture, movement of the child, and the maturation of the body's mechanisms and systems. In view of this, it is necessary to assess the motor development of children in a quilombola community, as it allows professionals in the field to investigate aspects and characteristics related to the development of this public. The aim of this study was to analyze the motor profile of quilombola children from Tiningú, Santarém/PA. The methodology is a descriptive and exploratory research with a quantitative and qualitative character. The sample consisted of 18 children of both sexes. The data collection tool was the child EDM test battery proposed by Rosa Neto (2002), it was necessary to divide into 2 days of data collection. Data underwent simple descriptive statistical analysis in Microsoft Office Excel 2010 and BioEstat version 5.0. The results, there are the classifications of the MGQ of the 18 children, where 11 children obtained $61.11 \%$, being in the average normal classification, 2 children reached $11.11 \%$, being compatible with the CI, and 5 children reached $27.78 \%$, being normal low. None had higher, lower and much lower motor age. It was concluded, from the results obtained, that the motor development of the evaluated children is within the parameters of normality, that is, compatible with the
\end{abstract}


chronological age in which they found themselves. Therefore, there were 2 groups of significant response, children who were above and below normality.

Keywords: Motor development; Play; Third childhood.

\section{Resumen}

El análisis del desarrollo motor es fundamental para establecer cambios en la conducta relacionados con la edad, el crecimiento, la postura, el movimiento del niño y la maduración de los mecanismos y sistemas corporales. Ante esto, es necesario evaluar el desarrollo motor de los niños en una comunidad quilombola, ya que permite a los profesionales en la materia investigar aspectos y características relacionados con el desarrollo de este público. El objetivo de este estudio fue analizar el perfil motor de niños quilombolas de Tiningú, Santarém / PA. La metodología es una investigación descriptiva y exploratoria de carácter cuantitativo y cualitativo. La muestra estuvo formada por 18 niños de ambos sexos. La herramienta de recolección de datos fue la batería de prueba de EDM infantil propuesta por Rosa Neto (2002), fue necesario dividirla en 2 días de recolección de datos. Los datos se sometieron a análisis estadístico descriptivo simple en Microsoft Office Excel 2010 y BioEstat versión 5.0. Los resultados, están las clasificaciones del MGQ de los 18 niños, donde 11 niños obtuvieron 61,11\%, estando en la clasificación promedio normal, 2 niños alcanzaron $11,11 \%$, siendo compatible con el IC, y 5 niños alcanzaron $27,78 \%$, siendo normal bajo. Ninguno tenía una edad motora mayor, menor o mucho menor. Se concluyó, a partir de los resultados obtenidos, que el desarrollo motor de los niños evaluados se encuentra dentro de los parámetros de normalidad, es decir, compatible con la edad cronológica en la que se encontraban. Por lo tanto, hubo 2 grupos de respuesta significativa, niños que estaban por encima y por debajo de la normalidad.

Palabras clave: Desarrollo motor; Juego; Tercera infancia.

\section{Introdução}

O desenvolvimento motor infantil é o processamento contínuo referente à idade cronológica, no qual a criança passa a adquirir inúmeras habilidades motoras, as quais seguem de movimentos fáceis desordenados para movimentos organizados. O processo de desenvolvimento acontece de forma dinâmica, sendo possível ser formado a partir de numerosos estímulos externos. Compreende-se que a princípio a origem e seu controle decorrem de uma direção céfalo-caudal e proximal distal, no entanto esse processo não se mostra de forma clara, envolvendo etapas de equilíbrios e desequilíbrios.

De acordo com Leite (2017) o desenvolvimento motor humano e divido em quatro fases: fase motora reflexa, fase de movimento rudimentares, fase de movimento fundamentais e fase de movimento especializados.

Carvalho (2011) afirma que, através desses processos adquiridos, a criança desenvolve habilidade no domínio motor, seja ela grosseira, fina, cognitiva ou emocional. Nesse aspecto, o desenvolvimento motor pode intervir no seguimento social, emocional e mental.

Ainda nessa perspectiva Mendes et al. (2015) afirmam que, quando acontece o atraso do desenvolvimento neuropsicomotor infantil, diversos aspectos podem estar relacionados ao problema, entre os quais estão a concepção, a gestação, o parto, a subnutrição, aspectos genéticos e agravos neurológicos. Nesse sentindo, os testes de avaliação são uma ferramenta muito utilizada em crianças com atraso no desenvolvimento motor, no qual se incluem várias mudanças, sendo elas a força muscular, motricidade fina, motricidade ampla e a fala, ou é feita avaliação abrangente das capacidades funcionais, além da escala de desenvolvimento motor (Rosa Neto et al., 2013).

Dessa forma, destaca-se a importância do desenvolvimento motor infantil, em que se estabelecem mudanças no comportamento referentes à idade, ao crescimento, à postura, ao movimento da criança e à maturação dos mecanismos e sistemas do organismo. Como a criança tem a sua individualidade moldada pelo ambiente no qual está inserida ou pela cultura que foi passada, isso pode influenciar na velocidade do processo de desenvolvimento. Neste sentido, Ramanholo et al. destacam que em caso sejam suprimidos estes estímulos a criança poderá crescer com déficit motriz e terá dificuldade na fase onde acontece o aperfeiçoamento das capacidades motoras. 
Segundo Teixeira (2017) o brincar e um excelente meio de impulsiona o desenvolvimento infantil, influenciando diretamente na construção de sua identidade e na autonomia da criança, que desenvolvem suas capacidades importantes conforme o raciocínio logico, memória, atenção e a interação.

Bispo et al (2020) destacaram que as brincadeiras e os jogos preferidos pelas crianças são, as brincadeiras lúdicas e culturas, jogos simbólicos e com regras, sendo que os jogos com regras são as escolhidas. Segundo Pontes e Magalhães (2003) esses tipos de jogo estimula as crianças a um ensaio para sua vida adulta respeitando as regras da sociedade.

O desenvolvimento motor na fase pré-escolar dessas crianças foi muito complexo, em razão da estrutura escolar, do saneamento básico e da falta de equipe multidisciplinar. Apesar de todos esses aspectos, as crianças dessa comunidade obtiveram uma relação muito íntima com a natureza, o que pode contribuir de forma positiva para seu desenvolvimento motor, através do brincar ao ar livre se relacionando com outras crianças.

Fernandes et al (2018) acredita-se que os escolares quilombolas, por morarem na zona rural, têm maiores opções de lazer ativo, desfrutando de experiências motoras adequadas, apesar de não apresentarem diferenças nas pesquisas com crianças não quilombolas.

Portanto, faz-se necessária a avaliação do desenvolvimento motor em crianças em comunidade quilombola, que se encontram em vulnerabilidade social, contribuindo para a melhoria da qualidade de vida e diminuição do número de morbidades.

Avaliar o desenvolvimento motor de crianças quilombolas se faz necessário para comparar e relacionar a somatória idade motora com a idade cronológica.

Este estudo teve o propósito de avaliar o perfil motor de crianças de uma comunidade quilombola de Santarém no estado do Pará e a finalidade de responder a seguinte problemática: como se apresenta o desenvolvimento motor das crianças de terceira infância na comunidade quilombola de Tiningú?

\section{Metodologia}

O método científico começa na observação organizada de fato, da realização de experiências, das deduções lógicas e da comprovação científica dos resultados adquiridos. Por tanto, é o caminho que se deve adotar para levar a formulação de uma teoria científica (Pereira et al, 2018).

Esta se trata de uma pesquisa descritiva e exploratória com caráter quantitativo e qualitativo. Ocorreu na comunidade quilombola de Tiningú, localizada na região rural oeste do Pará, próximo à cidade de Santarém. A amostra foi composta por 18 crianças da terceira infância de 7 a 11 anos de idade de ambos os sexos reconhecidas como quilombolas. A ferramenta de coleta dos dados foi a bateria de testes de EDM infantil proposta por Rosa Neto (2002), que foi necessário dividir em duas etapas, em dois dias, uma para recrutamento das crianças e início da coleta e a outra para finalizar a pesquisa e melhor coleta de dados. Os dados da pesquisa passaram por análises estatísticas descritivas simples no Microsoft Office Excel 2010 e BioEstat versão 5.0.

\section{Resultados}

De acordo com o objetivo de traçar o perfil do desenvolvimento motor de dezoito crianças de 7 a 11 anos de idade da comunidade de Tiningú, os resultados a seguir apresentam a análise descritiva das variáveis idade cronológica (IC), idade motora (IMG) e quociente motor referente às áreas da motricidade, tais como motricidade fina, motricidade global, equilíbrio, esquema corporal, organização espacial, organização temporal (QM1, QM2, QM3, QM4, QM5 e QM6) e o quociente motor geral (QMG). 
Tabela 1 - Perfil motor geral das crianças.

\begin{tabular}{|c|c|c|c|c|}
\hline Variáveis & Média & $\begin{array}{c}\text { Desvio } \\
\text { Padrão }\end{array}$ & $\begin{array}{c}\text { Valor } \\
\text { Mínimo }\end{array}$ & $\begin{array}{c}\text { Valor } \\
\text { Máximo }\end{array}$ \\
\hline IC & 111,44 & 19,33 & 84 & 132 \\
\hline IMG & 107,72 & 18,41 & 76 & 128 \\
\hline Quociente Motor Geral (QMG) & 96,92 & 7,34 & 87,30 & 108,20 \\
\hline Motricidade Fina (QM1) & 103,31 & 6,38 & 90,90 & 112,50 \\
\hline Motricidade Global (QM2) & 105,16 & 9,22 & 90 & 125,60 \\
\hline Equilíbrio (QM3) & 103,12 & 15,55 & 81,80 & 150 \\
\hline Esquema Corporal (QM4) & 89,98 & 14,20 & 63,60 & 110 \\
\hline Organização Espacial (QM5) & 87,48 & 13,13 & 50 & 106,40 \\
\hline Organização Temporal (QM6) & 92,46 & 18,85 & 60 & 137,50 \\
\hline
\end{tabular}

Fonte: Autores (2019).

Na Tabela 1, mostra-se de maneira linear o perfil motor das idades motoras próprias e gerais em meses, desse modo compreendeu-se que as idades motoras específicas alcançaram média de resultados dentro do esperado. Na idade cronológica (IC) a média foi de 111.44 meses, um desvio padrão de 19,33, valor mínimo e máximo de 84 e 132 respectivamente, classificado em normal alto. Na idade motora geral (IMG) a média foi de 107.72 meses, seu desvio padrão de 18,41, valor mínimo e máximo de 76 e 128 respectivamente, classificando-se de normal médio e estando 3 meses abaixo da idade cronológica.

O quociente motor geral (QMC) foi de 96.92, classificado como normal médio. No teste de motricidade fina, os participantes da amostra apresentaram uma média de 103,31 meses, um desvio padrão de 6,38, um valor mínimo e máximo de 90,90 e 112,50 respectivamente, tendo sido classificados como normal médio. Na coordenação global a média foi de 105,16 meses, apresentando um desvio padrão de 9,22 e valores mínimo e máximo de 90 e 125,60 respectivamente, correspondendo a um nível normal médio de aptidão motora nesta área motora. Quanto ao equilíbrio, atingiu-se uma média de 103,12 meses, apresentando um desvio padrão de 15,55 e valores mínimo e máximo de 81,80 e 150 respectivamente, classificando os participantes como normal médio.

O teste de esquema corporal obteve uma média de 89,98 meses, apresentando um desvio padrão de 14,20, valores mínimo e máximo de 63,60 e 110 respectivamente, atingindo a amostra a classificação de normal baixo. Com relação à organização espacial, as crianças atingiram uma média de 87,48 meses, apresentando um desvio padrão de 13,13 e valor mínimo de 50 e máximo de 106,40 pontos, obtendo uma classificação normal baixo. No teste de organização temporal, os participantes atingiram uma média de 92,46 meses, com valores mínimo e máximo de 60 e 137,50 respectivamente e um desvio padrão de 18,85, classificando-os no nível normal médio. 
Tabela 2 - Frequência da classificação do QMG.

\begin{tabular}{ccc}
\hline Classificação & $\mathbf{N}=$ & $\%$ \\
\hline Superior & 0 & 0 \\
Normal Alto & 2 & 11,11 \\
Normal médio & 11 & 61,11 \\
Normal baixo & 5 & 27,78 \\
Inferior & 0 & 0 \\
Muito inferior & 0 & 0 \\
TOTAL & 18 & 100,00 \\
\hline
\end{tabular}

Fonte: Autores (2019).

Na Tabela 2 apresentam as classificações da QMG das 18 crianças avaliadas, onde 11 crianças obtiveram $61.11 \%$ estando na classificação normal médio, e 2 crianças alcançaram $11.11 \%$ apresentam-se compatível com IC, e 5 crianças atingiu 27,78\% mostrando está em normal baixo. E nenhuma apresentou Idade Motora superior, inferior e muito inferior.

Tabela 3 - Classificação do QMG e seus QM das respectivas áreas motoras de 5 crianças.

\begin{tabular}{|c|c|c|c|c|c|c|c|}
\hline \multicolumn{2}{|c|}{ CRIANÇAS } & \multicolumn{5}{|c|}{ RESULTADOS DAS ÁREAS DA MOTRICIDADE (QM) } & \multirow[b]{2}{*}{ QM6 } \\
\hline $\mathbf{N}^{\circ}(\mathbf{Q M})$ & $\begin{array}{c}\text { Resultado } \\
\text { QMG }\end{array}$ & QM1 & QM2 & QM3 & QM4 & QM5 & \\
\hline $1(89.4)$ & Normal Baixo & $\begin{array}{l}\text { Normal } \\
\text { médio }\end{array}$ & $\begin{array}{l}\text { Normal } \\
\text { médio }\end{array}$ & $\begin{array}{c}\text { Normal } \\
\text { médio }\end{array}$ & $\begin{array}{l}\text { Muito } \\
\text { inferior }\end{array}$ & $\begin{array}{c}\text { Normal } \\
\text { baixo }\end{array}$ & $\begin{array}{c}\text { Normal } \\
\text { médio }\end{array}$ \\
\hline $2(87.5)$ & Normal Baixo & $\begin{array}{c}\text { Normal } \\
\text { alto }\end{array}$ & $\begin{array}{l}\text { Normal } \\
\text { médio }\end{array}$ & $\begin{array}{c}\text { Normal } \\
\text { médio }\end{array}$ & $\begin{array}{c}\text { Normal } \\
\text { médio }\end{array}$ & $\begin{array}{l}\text { Muito } \\
\text { inferior }\end{array}$ & $\begin{array}{l}\text { Muito } \\
\text { inferior }\end{array}$ \\
\hline $3(89.4)$ & Normal Baixo & $\begin{array}{c}\text { Normal } \\
\text { médio }\end{array}$ & $\begin{array}{c}\text { Normal } \\
\text { médio }\end{array}$ & $\begin{array}{c}\text { Normal } \\
\text { baixo }\end{array}$ & $\begin{array}{c}\text { Normal } \\
\text { médio }\end{array}$ & $\begin{array}{c}\text { Normal } \\
\text { baixo }\end{array}$ & $\begin{array}{c}\text { Normal } \\
\text { baixo }\end{array}$ \\
\hline $4(89.4)$ & Normal Baixo & $\begin{array}{c}\text { Normal } \\
\text { médio }\end{array}$ & $\begin{array}{l}\text { Normal } \\
\text { médio }\end{array}$ & $\begin{array}{c}\text { Normal } \\
\text { médio }\end{array}$ & $\begin{array}{l}\text { Muito } \\
\text { inferior }\end{array}$ & $\begin{array}{l}\text { Normal } \\
\text { médio }\end{array}$ & $\begin{array}{c}\text { Normal } \\
\text { médio }\end{array}$ \\
\hline $5(87.3)$ & Normal Baixo & $\begin{array}{c}\text { Normal } \\
\text { médio }\end{array}$ & $\begin{array}{c}\text { Normal } \\
\text { médio }\end{array}$ & $\begin{array}{c}\text { Normal } \\
\text { médio }\end{array}$ & $\begin{array}{c}\text { Normal } \\
\text { baixo }\end{array}$ & $\begin{array}{c}\text { Normal } \\
\text { baixo }\end{array}$ & $\begin{array}{l}\text { Muito } \\
\text { inferior }\end{array}$ \\
\hline
\end{tabular}

Fonte: Autores (2019).

$\mathrm{Na}$ Tabela 3, apresentam os resultados das áreas da motricidade de cinco crianças, na qual a primeira criança apresenta o QMG normal baixo e motricidade fina, global, equilíbrio e organização temporal classificados como normal médio, enquanto o esquema corporal mostra-se muito inferior e a organização espacial está normal baixo.

A segunda criança identifica-se com QMG normal baixo e motricidade fina normal alta, já a motricidade global, o equilíbrio e o esquema corporal apresentaram-se como normal médio, ao mesmo tempo que a organização espacial e a temporal foram de muito inferior. Na terceira criança o QMG foi normal baixo e as motricidades fina e global e o esquema corporal se classificaram como normal médio, já o equilíbrio e a organização espacial e temporal mostraram-se normal baixo.

A quarta criança mostrou QMG normal baixo e motricidades fina e global, equilíbrio e organização espacial e temporal classificados como normal médio, em contrapartida o esquema corporal mostrou-se muito inferior. A quinta e última criança mostrou também QMG normal baixo, motricidades fina e global e equilíbrio classificados como normal médio, enquanto o 
esquema corporal e organização espacial mostram-se normal baixo, diferente da organização espacial, que se identificou muito inferior.

Com relação à Tabela 3, foram observadas três crianças com classificações na QM4, QM5 e QM6 alteradas para muito inferior em níveis de risco muito graves em comparação com a idade cronológica (IC). Com isso, analisou-se que três crianças, ao realizar as baterias com testes de esquema corporal, organização espacial e temporal, obtiveram resultados muito inferiores referentes à idade cronológica e às outras áreas da motricidade. Essas crianças tiveram um déficit na consciência corporal, a qual é de fundamental importância para criança ter um bom desenvolvimento, que presume uma evolução da motricidade, dando compreensões também para organizações espacial e temporal.

Tabela 4 - Classificação do QMG e seus QM das respectivas áreas motoras de 2 crianças.

\begin{tabular}{|c|c|c|c|c|c|c|c|}
\hline \multicolumn{2}{|c|}{ CRIANÇAS } & \multicolumn{5}{|c|}{ RESULTADOS DAS ÁREAS DA MOTRICIDADE (QM) } & \multirow[b]{2}{*}{ QM6 } \\
\hline$\overline{\mathbf{N}^{\circ}(\mathbf{Q M G})}$ & $\begin{array}{c}\text { Resultado } \\
\text { QMG }\end{array}$ & QM1 & QM2 & QM3 & QM4 & QM5 & \\
\hline $1(108,2)$ & Normal Alto & $\begin{array}{c}\text { Normal } \\
\text { alto }\end{array}$ & $\begin{array}{c}\text { Normal } \\
\text { alto }\end{array}$ & $\begin{array}{c}\text { Normal } \\
\text { alto }\end{array}$ & $\begin{array}{c}\text { Normal } \\
\text { médio }\end{array}$ & $\begin{array}{c}\text { Normal } \\
\text { médio }\end{array}$ & Superior \\
\hline $2(104,1)$ & Normal Alto & $\begin{array}{c}\text { Normal } \\
\text { alto }\end{array}$ & $\begin{array}{c}\text { Normal } \\
\text { alto }\end{array}$ & $\begin{array}{c}\text { Normal } \\
\text { alto }\end{array}$ & $\begin{array}{c}\text { Normal } \\
\text { médio }\end{array}$ & $\begin{array}{c}\text { Normal } \\
\text { médio }\end{array}$ & $\begin{array}{c}\text { Normal } \\
\text { médio }\end{array}$ \\
\hline
\end{tabular}

Fonte: Autores (2019).

$\mathrm{Na}$ Tabela 4, apresentam as classificações do QMG e seus QM das respectivas áreas motoras de duas crianças, podendo ser observado que as duas crianças testadas se classificam como normal alto, sendo que a primeira apresentou QMG de 108,2 meses e a segunda QMG de 104,1 meses, sendo que nos resultados das áreas da motricidade (QM) houve diferença na organização temporal (QM6), em que a primeira obteve a classificação superior e a segunda apresentou normal médio, sendo que nem uma apresenta risco.

\section{Discussão}

Quanto ao demonstrado na Tabela 1, Rosa Neto (2015) afirma que esses resultados são o que se espera para amostras de crianças que não apresentam alterações sensoriais, neurológicas, mentais ou físicas. Silveira (2005 apud Costa; Silva, 2009, p. 59) concluem que "com o aumento da idade cronológica os indivíduos são capazes de realizar funções mais complexas".

De acordo com essa tabela, a menor pontuação foi na organização espacial (QM5), cujo valor foi de 87,48 meses com relação à idade cronológica, classificando-se como normal baixo, então pode-se compreender que as crianças tiveram dificuldade e que há uma deficiência motora na organização espacial. Do mesmo modo, o estudo de Amaro et al. (2008) também encontrou classificação muito inferior nas áreas da organização espacial e temporal em crianças com dificuldades na aprendizagem.

Corroborando Rosa Neto (2015), o desenvolvimento mental da criança evolui gradualmente na aquisição e conservação das bases de distância, superfície, forma, tamanho, espessura, perspectivas e coordenadas, que determinam as probabilidades de orientação e de organização do espaço em que vivem, sendo assim a criança que tem dificuldade nessa competência não será capaz de compreender de forma progressiva.

Referente à Tabela 2, o estudo de Rosa Neto et al. (2010) apresentou o mesmo índice, mas com valores diferentes, a finalidade era avaliar o desenvolvimento motor de crianças com indicadores de dificuldades na aprendizagem escolar, os pesquisadores verificaram que, de acordo com a classificação da Escala de Desenvolvimento Motor, 74,3\% dos escolares apresentaram índice de normal médio a normal baixo com média do QMG de 89,48 (normal baixo). Do total de escolares 
avaliados, $87,1 \%(\mathrm{n}=27)$ apresentou média de 16 meses de atraso motor, informações essas que se comparam aos achados do presente estudo.

Fonseca (1983), Ferreira et al. (2006), Silva et al. (2006) e Poeta e Rosa Neto (2007) realizaram estudos em que todas as crianças apresentaram grandes dificuldades motoras. De certa forma, essas crianças também apresentaram dificuldades na aprendizagem, que já era de se aguardar em virtude de que estes estudos estão relacionados com o desenvolvimento motor e rendimento escolar e demonstram uma forte relevância estatística entre o que a criança é capacitada de entender (cognitivo) e o que é capacitada de fazer (motor).

Com relação à Tabela 3, foram observadas três crianças com classificações na QM4, QM5 e QM6 alteradas para muito inferior em níveis de risco muito graves em comparação com a idade cronológica (IC). Com isso, analisou-se que três crianças, ao realizar as baterias com testes de esquema corporal, organização espacial e temporal, obtiveram resultados muito inferiores referentes à idade cronológica e às outras áreas da motricidade. Essas crianças tiveram um déficit na consciência corporal, a qual é de fundamental importância para a criança ter um bom desenvolvimento, que presume uma evolução da motricidade, dando compreensões também para as organizações espacial e temporal.

Esse resultado corrobora o estudo de Medina, Rosa e Marques (2006), que revelou atraso no desenvolvimento motor das crianças na classificação da faixa etária de 9 a 10 anos de idade, na motricidade organização temporal, apresentando déficit, não alcançando a idade motora esperada. Esses resultados propõem uma progressão na incidência de atraso no desenvolvimento da organização temporal das crianças com dificuldades de aprendizagem conforme aumenta a idade cronológica.

Em contrapartida, Costa e Silva (2009) apresentaram no seu estudo que o único resultado encontrado de melhoria foi no esquema corporal, no qual o grupo mostrou uma grande evolução motora. Com isso, compreende-se que as quantidades e qualidades de estímulos recebidos nos estudos foram eficientes.

O estudo de Negrine (1986) leva a compreender que, com relação à organização espacial, há certa dificuldade na coleta das informações e na avaliação da relação física entre o corpo da criança e o ambiente por meio dos seus sentidos.

Quanto ao apresentado na Tabela 4, Medina, Rosa e Marques (2006) mostraram que na classificação geral para o desenvolvimento da organização temporal, de acordo com seu estudo, a maior parte de sua coleta (53\%) mostrou idade motora inferior à idade cronológica, confirmando que a maioria das crianças tiveram dificuldades de aprendizagem, da mesma forma apresentando déficit no desenvolvimento da organização temporal.

\section{Conclusão}

A partir dos resultados obtidos, foi possível constatar que o desenvolvimento motor dos avaliados encontra-se dentro dos parâmetros da normalidade, ou seja, compatíveis com a idade cronológica em que se encontravam. Porém, notaram-se dois achados significativos dentro dessa normalidade, crianças que se apresentaram acima da normalidade e crianças que se apresentaram abaixo da normalidade, sendo que duas crianças se apresentaram acima da média de normalidade no que tange à motricidade fina, global e equilíbrio.

Por outro lado, cinco crianças se destacaram abaixo da média de normalidade nas fases da motricidade esquema corporal, organização espacial e temporal. Portanto, tornam-se necessárias futuras intervenções e estímulos nessas áreas da motricidade na comunidade quilombola para que se evite atraso no desenvolvimento motor.

Diante do exposto, sugere-se que sejam realizadas novas pesquisas sobre a temática, tendo em vista a importância de abordar sobre a avaliação e desenvolvimento motor em crianças quilombolas, levando em consideração que este público tem menor acesso a assistência em saúde, agregando assim mais conhecimento para toda sociedade acadêmica e profissional que tenha interesse pela temática. 


\section{Agradecimentos}

Primeiro agradecemos a Deus por conseguir concluir mais uma etapa em nossas vidas. Segundo, agradecemos nossos familiares, que sempre nos apoiaram e nos incentivaram durante a árdua jornada acadêmica, também lembrar daqueles familiares que hoje não estão entre nós, mas que nos deram forças para realizar o sonho da graduação, que antes parecia distante da nossa realidade. Agradecemos o nosso orientador por, sempre, ser prestativo e presente, por nos guiar e apoiar nossas decisões. Tê-lo como orientador foi muito além da relação de professor e acadêmicas, pois tornou-se amigo e referência profissional. A parceria selada entre nossos amigos de graduação durante todos esses anos, com certeza, ficará na memória: os risos, discussões, lágrimas e as confraternizações. Por fim, é satisfatório perceber que apesar de tudo que passamos a graduação só nos fez mais fortes e persistente em nossos objetivos.

\section{Referências}

Amaro, K. N., Brusamarello, S., Campos, F. C. G., Corazza, T. D. M., \& Xavier, R. F. C. (2008). Análise da organização temporal em escolares com dificuldade de aprendizagem. Revista efdeportes.com, 13(127).

Bispo Leandro, C. C., Bispo Barbosa, L. \& Salazar, L. O. B. Inventário dos jogos e brincadeiras: a manifestação da cultura lúdica infantil. 5 (1), 500-522. https://periodicos.ifal.edu.br/diversitas_journal/

Carvalho, M. V. P. (2011). O desenvolvimento motor normal da criança de 0 à 1 ano: orientações para pais e cuidadores. Dissertação (Mestrado Profissional em Ensino em Ciências da Saúde e do Meio Ambiente) - Fundação Oswaldo Aranha, Centro Universitário de Volta Redonda. https://sites.unifoa.edu.br/portal_ensino/mestrado/mecsma/arquivos/37.pdf.

Costa, R. M., \& Silva, E. A. A. (2009). Escala de desenvolvimento motor de rosa neto: estudo longitudinal em uma escola da rede particular de ensino de Cuiabá - MT. Revista eletrônica do Univag, 4. http://periodicos.univag.com.br/index.php/CONNECTIONLINE/article/view/133.

Fernandes, J. L., Santiago, M. L. E., Lima, A. C. G., Nascimento, E. S. \& Moura, T. N. B. (2018). Desempenho motor de escolares quilombolas entre 8 e 10 anos. Revista Brasileira de Segurança Pública. 10.22278/2318-2660.2018.v42.n4.a2752. https://rbsp.sesab.ba.gov.br/index.php/rbsp/article/dow nload/2752/2593?inline $=1$.

Ferreira, L. F., Nascimento, R. O., Apolinário, M. R. \& Freudenheim, A. M. (2006). Desordem da coordenação do desenvolvimento. Motriz, 12(3), 283-292, set./dez. http://www.periodicos.rc.biblioteca.unesp.br/index.php/motriz/article/view/422/748.

Fonseca, V. Desenvolvimento Psicomotor e Aprendizagem. (1983). Editora Martins Fontes.

Leite, F. S. Desenvolvimento psicomotor de crianças de 4 a 6 anos de escola particular em lima campos -MA. (2017). Revista Rebai. 1(1), 1-23.

Medina, J., Rosa, G. K. B., \& Marques, I. (2006). Desenvolvimento da organização temporal de criança com dificuldade aprendizagem. Revista da Educação Física, 17(1), 107-116, 1. https://www.researchgate.net/publication/283529760_Desenvolvimento_da_organizacao_tem poral_de_criancas_com_dificul dades_de_aprendizagem.

Mendes, M. V. S., Cavalcante, S. A., Oliveira, E. F., Pinto, D. M. R., Barbosa, T. S. M. \& Camargo, C. L. (2015). Crianças com retardo do desenvolvimento neuropsicomotor: musicoterapia promovendo qualidade de vida. Revista Brasileira Enfermagem, 68(5). https://doi.org/10.1590/0034-7167.2015680505i. https://www.scielo.br/j/reben/a/yLNkGB8krCPw6SLKnNVwYTK/?lang=pt.

Negrine, A. Educação psicomotora: a lateralidade e a orientação espacial. Editora Pallotti, 1986.

Pereira, A. S., Shitsuka, D. M., Parreira, F. J. \& Shitsuka, R. (2018). Ciência e conhecimento científico. Metodologia da pesquisa científica.

Poeta, L. S., \& Rosa Neto, F. (2007). Motor assessment in school with indicators of the attention deficit/hyperactivity disorder. Rev. Neur. 44(3). https://neurologia.com/articulo/2005663/eng.

Pontes, F. A. R., \& Magalhães, C. M. C. (2003). A transmissão da cultura da brincadeira: algumas possibilidades de investigação. Psicologia: reflexão e crítica. Revista de Periódicos Eletrônicos em Psicologia. 16(1), 117-124.

Romanholo, R. A., Heydrich, V., Almeida, A. M., Coelho, E. M. \& Carvalhal, M. I. (2012). Análise da relação entre a maturação biológica e estresse na coordenação motora grossa em escolares de 5 a 10 anos. Revista Brasileira Ciência Movimento. 20(2):91-7. 10.18511/rbcm.v20i2.3262.

Rosa Neto, F. (2015). Manual de Intervenção Motora: educação infantil, fundamental e educação especial. Florianópolis: Editora Dioesc.

Rosa Neto, F. Manual de avaliação motora. (2002). Editora Artmed.

Rosa Neto, F., Santos, A. N. M., Xavier, R. F. C. \& Amaro, K. N. (2010). A importância da avaliação motora em escolares: análise da confiabilidade da escala de desenvolvimento motor. Revista Brasileira Cineantropom Desempenho Humano, 12(6), 422-427.

Rosa Neto, F., Xavier, R. F. C., Santos, A. P. M. dos., Amaro, K. N., Florêncio, R. \& Poeta, L. S. (2013). A lateralidade cruzada e o desempenho da leitura e escrita em escolares. Revista CEFAC, 15(4), 864-872. https://doi.org/10.1590/S1516-18462013000400015. https://www.scielo.br/j/rcefac/a/WfKW8Mw7Pwwd 9KN5sCtRdTH/?lang=pt. 
Research, Society and Development, v. 10, n. 13, e523101321860, 2021

(CC BY 4.0) | ISSN 2525-3409 | DOI: http://dx.doi.org/10.33448/rsd-v10i13.21860

Silva, C. A., Rosa Neto, F., Almeida, G. M. F., Amaro, K. N., \& Beckhäuser, M. (2007). A importância da avaliação motora em escolares. Revista Íbero Americana de Psicomotridad y Técnicas Corporales, (26), 137-144. https://dialnet.unirioja.es/servlet/articulo?codigo=3620837.

Teixeira, C. C. S. (2017). A importância da brincadeira no desenvolvimento cognitivo infantil. 2016. Id on Line Revista Multidisciplinar de psicologia. 10(33), 94-102. http://idonline.emnuvens.com.br/id. 